\title{
GALILEO UVS RESULTS AND CASSINI PREVIEW
}

\author{
L.W. ESPOSITO, C.A. BARTH, A.R. HENDRIX, C.W. HORD AND A.I.F. STEWART \\ Laboratory for Atmospheric and Space Physics \\ University of Colorado \\ Boulder, CO 80309
}

AND

J.M. AJELLO AND R.A. WEST

Jet Propulsion Laboratory

Pasadena, CA 91109

\begin{abstract}
UVS results from Jupiter show the Io torus colder and brighter than the Voyager observations. Aurora near Jupiter's poles emit $1-4 \mathrm{MR}$ from altitudes of $300-400 \mathrm{~km}$. Ganymede and Callisto have extended hydrogen exospheres. The near-UV reflectance of the Galilean satellites shows $\mathrm{SO}_{2}$, magnetospheric alteration, and $\mathrm{O}_{3}$. Remote sensing of Jupiter's stratospheric haze determines that it thickens and darkens towards higher latitudes.
\end{abstract}

\section{Io Torus}

Observations of the Io plasma torus by the Galileo Extreme Ultraviolet Spectrometer (EUV) from Jupiter approach and G1, C3, E4, E6, G7, and G8 are analyzed by Stewart et al. (1997). The EUV spectra all reflect a noticeably lower electron temperature than do the discovery spectra by the Voyager UVS instruments. Figure 1 shows a comparison between the observed Galileo spectrum and the model based on Voyager data. The Galileo instrument also measures higher luminosity than Voyager, and from orbit to orbit there is a clear anti-correlation between temperature and luminosity. Galileo data also show the midnight ansa is brighter than the noon ansa by some tens of percent, and that the dusk ansa is brighter than the dawn ansa by a factor of two or more. Two possible states appear in the Galileo data: (1) the plasma torus is bright, cool, and dense or (2) it is $\operatorname{dim}$, warm, and tenuous.

\section{Jupiter Aurora}

In 1996, during the first four orbits of the Satellite tour, the Galileo Ultraviolet Spectrometer (UVS, $110-430 \mathrm{~nm}$ ) and Extreme Ultraviolet Spectrometer (EUV, $54-128 \mathrm{~nm}$ ) performed near simultaneous observations of the Jupiter aurora in both the south and north polar regions. These data were analyzed by Ajello et al. (1997). These observations were modeled to provide absolute surface brightness of the aurora from the $\mathrm{H}_{2}$ Rydberg Systems (B, B', B', C, D, D' $\rightarrow$ X band systems). The EUV, which is located on the spinning portion of the spacecraft, has an intrinsically small duty cycle on the auroral oval. Nonetheless, the EUV acquired high-quality spectra of the aurora because of the lower than expected radiation background along with increased radiation shielding relative to the Voyager UVS. Regression analysis of the emission model shows the EUV aurora $(80-120 \mathrm{~nm})$ is produced in a range of altitudes corresponding to the $\mathrm{H}_{2}$ slant column from 1016 to $1020 \mathrm{~cm}^{-2}$. Both the EUV and FUV spectra require hydrocarbon absorbers not yet included in current models. The FUV spectra show methane column abundance 1 to $5 \times 10^{16} \mathrm{~cm}^{-2}$. The $\mathrm{H}_{2}$ Rydberg Systems emit $1-4 \mathrm{MR}$ and H Lyman alpha $200-500 \mathrm{kR}$ for a $2000-\mathrm{km}$ wide oval. Both north and south aurora emit $10^{13}$ watts. Variability and morphology seen by Galileo is consistent with HST observations. 


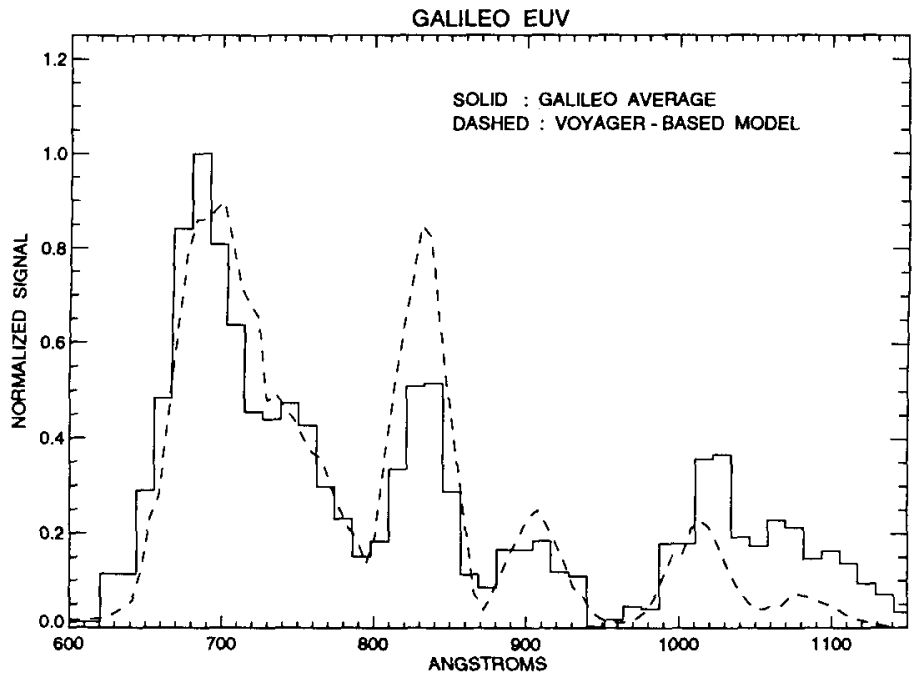

Figure 1. Galileo EUV observation of Io torus (solid line) compared to model based on Voyager data.

\section{Satellite Exospheres}

Atomic hydrogen Lyman alpha radiation $(121.6 \mathrm{~nm})$ has been measured in emission from the atmospheres of Ganymede and Callisto (Barth et al. 1997) by the Galileo UVS. The density directly above the surface of Ganymede is $1.5 \times 10^{4}$ atoms $\mathrm{cm}^{-3}$. The altitude distribution is consistent with a scale height that corresponds to a temperature of $450 \mathrm{~K}$. A possible source of the atomic hydrogen is photo-dissociation of water vapor subliming from the surface ice. In this model, the escape flux of atomic hydrogen is $7 \times 10^{8}$ atoms $\mathrm{cm}^{-2} \mathrm{sec}^{-1}$, corresponding to ice removal of $1 \mathrm{~nm} /$ year. The amount of oxygen accumulating on Ganymede should be comparable. This mechanism requires a substantial surface ice reservoir at $T=146 \mathrm{~K}$. Two other possible hydrogen atom source mechanisms are photo-desorption by solar photons and sputtering by Jupiter magnetospheric ions.

\section{Jupiter UV-Absorbing Haze}

Galileo UVS spectra taken on 6 September 1996 between 220 - $230 \mathrm{~nm}$ have been compared to HST WFPC2 images at $230 \mathrm{~nm}$ and $275 \mathrm{~nm}$ from 27 June 1997 (West et al. 1997). The Galileo data provide good spectral coverage and spatial resolution along the central meridian. The Hubble data have complete spatial coverage and good resolution at the two wavelengths imaged. Analysis shows that the high-latitude haze is concentrated between $3-10 \mathrm{mb}$. The mid-latitude haze may be deeper. The haze optical depth increases toward the poles, but not symmetrically in the North and South hemispheres. The aerosol scattering phase function is forward peaked, with moderate back-scattering. The single scattering albedo decreases with latitude and wavelength. The aerosol optical properties are an essential input for models of solar heating and stratospheric circulation.

\section{Cassini Preview}

The Cassini Ultraviolet Imaging Spectrograph (UVIS) is a 2-channel imaging spectrograph (Esposito et al. 1997) aboard the Cassini Orbiter. Its spectral range is from $55-190 \mathrm{~nm}$, with adjustable spectral resolution $0.2-0.5 \mathrm{~nm}$. The imaging capability is provided in one dimension along the slit with spatial resolution of $2 \mathrm{mrad}$. The optical axis is moved perpendicular to the slit to produce images. The Cassini Jupiter flyby is scheduled for 30 December 2000 at a distance of $139 \mathrm{R}_{J}$. The spectrometer will carry out comprehensive measurements near Jupiter by systematically scanning in a raster pattern parallel to Jupiter's equator. Expected results include the Jupiter atomic hydrogen distribution, maps of the Io torus, and low spatial resolution auroral and cloud spectroscopy. 
These results and later observations during Cassini's Saturn orbital tour can be directly compared to the Galileo UVS results.

\section{References}

Ajello, J. et al. (1997). J. Geophys. Res., (submitted).

Barth, C.A. et al. (1997) Geophys. Res. Lett., 24, pp. 2147-2150.

Esposito, L.W. et al. (1977) Space Science Revieus, (to appear).

Hendrix, A.R. (1997) The Galileo ultraviolet spectrometer: in-flight calibration and UV albedos of the Moon, Gaspra, Ida, and Europa. Ph.D. Dissertation, University of Colorado.

Stewart, A.I.F. et al. (1997) Paper presented at IAGA, August 1997.

West, R.A. et al. (1997) Paper presented at DPS, July 1997. 